\title{
Orientation and spectral properties of two stilbazolium merocyanine dyes in stretched and unstretched polyvinyl alcohol films
}

\author{
Alina Dudkowiak ${ }^{1 凶}$, Danuta Frąckowiak ${ }^{1}$, Ewa Teślak ${ }^{1}, Z^{2}$ gmunt Gryczyński \\ and Ignacy Gryczyński ${ }^{2}$ \\ ${ }^{1}$ Institute of Physics, Faculty of Technical Physics, Poznań University of Technology, Poznań, Poland; \\ ${ }^{2}$ Center for Commercialization of Fluorescence Technologies, Department of Molecular Biology and Immunology, \\ and Department of Cell Biology and Genetics, University of North Texas Health Science Center, Texas, USA
}

Received: 01 February, 2007; 19 July, 2007; accepted: 14 September, 2007

available on-line: 18 September, 2007

\begin{abstract}
Spectral properties (anisotropy coefficients calculated for absorption, emission and fluorescence decay time) of two stilbazolium merocyanine dyes have been determined to evaluate the applicability of these dyes as sensitizers in photodynamic therapy. The dyes were embedded in an anisotropic polymer matrix. Analysis of the emission decay components measured in polarized light provides information on the interactions of the dye molecules with the polymer matrix being a model of an anisotropic biological system. Different values of the emission anisotropies obtained from various polarized components of fluorescence decays have shown that the orientations of the dye molecules influence their interactions with the polymer. This means that differently oriented dye molecules located in biological systems should exhibit different interactions with membranes. The chain length and type of side groups attached as well as the salt form of the dye molecule were shown to influence the dye-polymer interactions and should be taken into account before the application of merocyanine dyes in medicine. These dyes seem to be promising optical sensors with spectral properties, including the calculated anisotropy coefficients, sensitive to the molecular environment, useful to study orientation and interaction with neighbouring molecules in biological membranes.
\end{abstract}

Keywords: stilbazolium merocyanine dyes, photodynamic therapy, anisotropic polymer matrix

\section{INTRODUCTION}

Stilbazolium merocyanine (Mero) dyes are promising candidates for application as sensitizers in photodynamic therapy (PDT) and in photodynamic diagnosis (PDD) as well as fluorescent markers in biological investigation (Gruda et al., 1987; Smith et al., 1991; O’Brien et al., 1992; Frąckowiak et al., 1995; Wiktorowicz et al., 1995; Lydaki et al., 1996). In PDT, a sensitizer has to exhibit strong efficiency in generation of very photochemically active triplet states (Staśkowiak et al., 2004; 2005). Therefore, the intersystem crossing (ISC) transition from the excited singlet state to the triplet state has to be efficient. This transition competes mainly with the fluorescence emission (Frąckowiak et al., 1990; Mishra et al., 2000). In PDD, the marker molecules have to be localized in the vicinity of cancerous cells and have to exhibit strong fluorescence (Wiktorowicz et al., 2004). Both Mero A (in a previous paper (Staśkowiak et al., 2004) marked as H) and Mero B (formerly - $\mathrm{U}^{*}$ ) (Fig. 1) were investigated by us recently (Staśkowiak et al., 2004, 2005). They differ in the molecular length as well as the types of side groups, and Mero B is in a salt form. It is known that such differences may influence the interaction of the dye with the surrounding environment (Frąckowiak et al., 1990), aggregation, its orientation in anisotropic media (Staśkowiak et al., 2005), and its photochemical properties (Mishra et al., 2000). In so-

Corresponding author: Alina Dudkowiak, Institute of Physics, Faculty of Technical Physics, Poznań University of Technology, Nieszawska 13a, 60-965 Poznań, Poland; e-mail: alina.dudkowiak@put.poznan.pl

Abbreviations: ET, energy transfer; ISC, intersystem crossing; Mero, merocyanine; PDD, photodynamic diagnosis; PDT, photodynamic therapy; PVA, polyvinyl alcohol; TMs, transition moments. 
lutions, Mero A and Mero B exhibit different spectral properties and both dyes show a very low yield of fluorescence (Staśkowiak et al., 2004), therefore they can be promising candidates for application in PDT but not for PDD. The investigated dyes dissolved in alcohol solution differ strongly in the yield of triplet states generation which is higher for Mero A than for Mero B (Staśkowiak et al., 2004). All spectral properties of dyes and natural pigment molecules depend strongly on the interactions with their surroundings (Bucci et al., 1992; Gryczyński et al., 1993; Frąckowiak et al., 2004; Bojarski et al., 2006). As a result, the spectral properties of dyes are different in cells and in solution (Staśkowiak et al., 2004; 2005). The pigment molecules attached to a biological or model membrane are usually oriented with respect to the membrane plane or polymer axis (Frąckowiak et al., 1990). In oriented systems the spectra of dye molecules exhibit higher anisotropies of absorption and emission than the average spectral anisotropies of molecules freely moving in solution or inside cells. The fractions of differently oriented dye molecules typically have different photochemical properties (Frąckowiak et al., 1990; 2004; Staśkowiak et al., 2005).

In the present paper we compare the spectral properties such as anisotropies of absorption and fluorescence, as well as decay times of emission obtained for Mero A and Mero B dyes (Fig. 1) located in isotropic (unstretched) and anisotropic (stretched) polymer (polyvinyl alcohol (PVA)) films. A comparison of the anisotropy coefficients obtained from the polarized absorption and emission spectra with those obtained from calculations for all the polarized components of the fluorescence decay of different decay times, brings information on the orientations of the different dye species and their interactions

Mero A

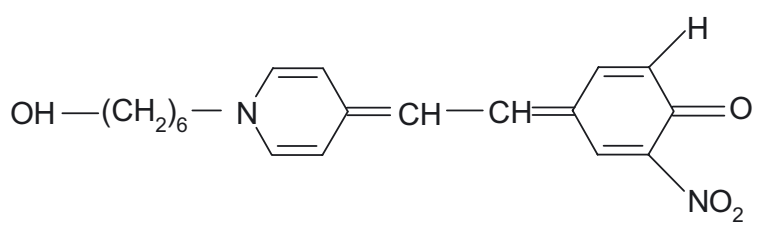

Mero B

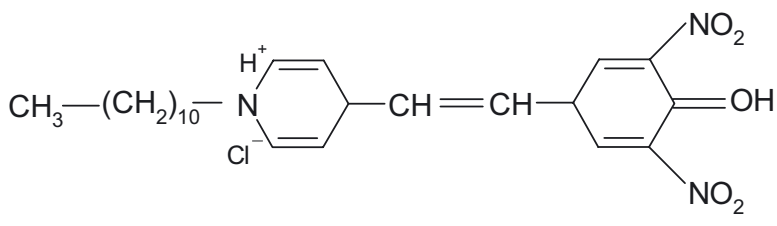

Figure 1. Molecular structure and notation of investigated stilbazolium merocyanines. with the polymer matrix. The fluorescence intensity decay profiles were analyzed using a multiexponential model (Lakowicz et al., 1991; 1999). We expected that such an analysis would give information about the groups of molecules differently interacting with the polymer matrix and therefore emitting fluorescence with different decay times and anisotropies. In the polarized emission spectra the contributions from such groups of molecules are averaged. A complex interrelation between the intensity and anisotropy of the fluorescence decay would be observed in the presence of fractions of dye molecules differently interacting with the polymer. The optical properties of these fractions should be dependent on the dyepolymer interactions, and therefore photoselection using polarized light should distinguish the fractions of dye molecules differently oriented with respect to the polymer. Film stretching usually causes a strong increase in the anisotropy of the dye emission. A stretched PVA film serves as an anisotropic model of a cell membrane (Bojarski et al., 2006). The dye molecules in such a matrix could be treated as a model of sensitizer molecules incorporated into biological membranes.

\section{MATERIALS AND METHODS}

The stilbazolium merocyanines (Fig. 1) were kindly provided by Dr. I. Gruda (Frąckowiak et al., 1990). The investigated compounds differ in the length of the $\left(\mathrm{CH}_{2}\right)$ chain and in the character of side groups. Additionally, Mero B is in a salt form.

PVA (from Aldrich) was used for preparation of isotropic and anisotropic films as previously described (Staśkowiak et al., 2004). Absorption spectra of the investigated sample were measured with a Varian Cary 4000 spectrophotometer, and fluorescence emission and fluorescence excitation spectra were measured with a Fluorescence Spectrofluorometer F4500 (Hitachi).

The fluorescence intensity decay profiles of the Mero dyes in isotropic and stretched PVA films were measured using a front-face configuration as described earlier (Lakowicz, 1999; Frąckowiak et al., 2004) with time-correlated single-photon-counting (FluoTime 200 fluorometer PicoQuant, Inc., Berlin, Germany). This instrument is equipped with a micro-channel plate (MCP) on the detection, and pulsed excitation sources (Laser Diodes: $375 \mathrm{~nm}$ and $475 \mathrm{~nm}$ and pulsed LED: blue providing $449 \mathrm{~nm}$, and green $-510 \mathrm{~nm}$ ). It is capable of resolving lifetimes that are below 50 ps for the laser diodes, and about 100 ps for LED excitation.

For stretched samples, two polarized absorption components of coloured and uncoloured polymer, $\mathrm{VV}$ and $\mathrm{VH}$, were measured $(\mathrm{V}$, vertical, par- 
allel to stretching direction; $\mathrm{H}$, horizontal) and the degree of orientation

$\mathrm{S}=(\mathrm{VV}-\mathrm{VH}) /(\mathrm{VV}+2 \mathrm{VH})=\left(3 \cos ^{2} \Theta-1\right) / 2$

where $\Theta$ is the angle between transition moments (TMs) of absorption and the stretching direction of the polymer film, was calculated. The first letter describes the polarization of incident light and the second letter indicates the position of the film axis.

The fluorescence was observed through a polarizer oriented vertically or horizontally $(\mathrm{V}$ or $\mathrm{H})$ relative to the stretching direction. The following four components were measured for the stretched films: VVV, VVH, VHH, VHV (first letter, polarization of the excitation light beam; middle, orientation of the stretching direction; the last one, polarization of the emission measured) and the coefficients of anisotropy (Eqns. 2-5) for the maxima of emission were calculated. For the unstretched (isotropic) films, the middle letter was 0 , therefore the components were marked as $\mathrm{VOV}$ or $\mathrm{VOH}$.

The four coefficients of anisotropy (Frąckowiak et al., 1990) were calculated from the emission spectra (Eqns. 2-5). The $r_{a}$ coefficient (Eqn. 2) describes the polarization of the emission from the molecules with their TMs of absorption at small angles with the direction of the film stretching, whereas $r_{b}$ (Eqn. 3 ) is related to the dye molecules with the TMs of emission at greater angles with respect to the film axis:

$\mathrm{r}_{\mathrm{a}}=(\mathrm{VVV}-\mathrm{VVH}) /(\mathrm{VVV}+2 \mathrm{VVH})$

$\mathrm{r}_{\mathrm{b}}=(\mathrm{VHV}-\mathrm{VHH}) /(\mathrm{VHV}+2 \mathrm{VHH})$

The $r_{c}$ parameter (Eqn. 4) describes the difference between the contributions to the parallel polarized emission from the molecules oriented in parallel and in perpendicular to the film axis, whereas $r_{d}$ (Eqn. 5) informs about the difference between the molecules oriented perpendicular and parallel with respect to the emission component observed.

$\mathrm{r}_{\mathrm{c}}=(\mathrm{VVV}-\mathrm{VHV}) /(\mathrm{VVV}+2 \mathrm{VHV})$

$\mathrm{r}_{\mathrm{d}}=(\mathrm{VVH}-\mathrm{VHH}) /(\mathrm{VVH}+2 \mathrm{VHH})$

There are several methods of analyzing fluorescence decays (Lakowicz et al., 1991; 1999). In the present investigation the data were fitted using the FluoFit software (PicoQuant v.4) to the multiexponential model:

$I(t)=\Sigma \alpha_{i} \exp \left(-t / \tau_{i}\right)$

where $\alpha_{i}$ is the amplitude of each decay component, $\tau_{i}$ is the fluorescence lifetime of each respective ith component. No assumptions are made as to the spectral shapes of components decaying with different times. The amplitude associated with the ith component of decay is given by:

$\alpha_{\mathrm{i}}=\left[\int_{0} \alpha_{\mathrm{i}}(\tau) \mathrm{d} \tau\right] /\left[\int_{0} \Sigma \alpha_{\mathrm{i}}(\tau) \mathrm{d} \tau\right]$

The fractional contribution of the ith component to the total emission is given by:

$\mathrm{f}_{\mathrm{i}}=\left[\int \alpha_{\mathrm{i}}(\tau) \tau \mathrm{d} \tau\right] /\left[\int \Sigma \alpha_{\mathrm{i}}(\tau) \tau \mathrm{d} \tau\right]$

The average lifetime can be characterized by two formulas: intensity-weighted (Eqn. 9) or amplitude-weighted (Eqn. 10) as follows:

$\tau_{\mathrm{AVI}}=\Sigma \mathrm{f}_{\mathrm{i}} \tau_{\mathrm{i}}$

$\tau_{\mathrm{AVA}}=\Sigma \alpha_{\mathrm{i}} \tau_{\mathrm{i}}$

Both values provide information useful for comparison of the sample properties. The fit of the intensity decay data to the multi-exponential model was based on nonlinear least square analysis. Each intensity decay was analyzed with $1,2,3 \ldots$ exponentials, and the reduced chi square $\left(\chi^{2}\right)$ values (Tables 2 and 3) were compared. If the goodness of fit was not improved with a higher number of components, a less complex model was selected.

For isotropic films, a more detailed information on the fractions of different dye orientations can be obtained from the coefficients $R_{a}$ and $R_{b}$, based on the $\mathrm{VOV}$ and $\mathrm{VOH}$ decay components of the emission measured and determined from the formula:

$\mathrm{R}_{\mathrm{a}}=[(\mathrm{VVV} / \mathrm{VOV})-(\mathrm{VVH} / \mathrm{VOH})] /[(\mathrm{VVV} / \mathrm{VOV})+$

$+2(\mathrm{VVH} / \mathrm{VOH})]$

$\mathrm{R}_{\mathrm{b}}=[(\mathrm{VHV} / \mathrm{VOV})-(\mathrm{VHH} / \mathrm{VOH})] /[\mathrm{VHV} / \mathrm{VOV})+$

$+2(\mathrm{VHH} / \mathrm{VOH})]$

$R_{a}$ is high when (in a given fraction of the dye) the TMs of absorption and emission are parallel to each other, and make small angles with the film axis, or when there is a large pool of well oriented molecules and the fraction of randomly oriented ones is small (Frąckowiak et al., 1990). The $R_{b}$ value describes the fluorescence anisotropy of the dye fraction with TMs of absorption and emission at large angles with the film axis, or with TMs with a broad distribution of angles to the film axis. This coefficient can be negative for molecules oriented at a small angle with respect to the film axis. The orientation distribution function for a uniaxially oriented system is defined by the angle between the long axis of the molecule and the preferred direction of orientation (Dalmolen et al., 1985). This function can be approximated by the following Legendre polynomials: 
$<\mathrm{P}_{2}>=\left[2+7 \mathrm{R}_{\mathrm{a}}-14 \mathrm{R}_{\mathrm{b}}+5 \mathrm{R}_{\mathrm{a}} \mathrm{R}_{\mathrm{b}}\right] /\left[23-14 \mathrm{R}_{\mathrm{a}}+\right.$

$\left.+R_{b}-10 R_{a} R_{b}\right]$

$<\mathrm{P}_{4}>=\left[-12+21 \mathrm{R}_{\mathrm{a}}+21 \mathrm{R}_{\mathrm{b}}-30 \mathrm{R}_{\mathrm{a}} \mathrm{R}_{\mathrm{b}}\right] /\left[23-14 \mathrm{R}_{\mathrm{a}}+\right.$

$\left.+R_{b}-10 R_{a} R_{b}\right]$

The value of $\left\langle\mathrm{P}_{2}\right\rangle$ gives the information about the orientation of molecules similar to that obtained from the absorption anisotropy, but for different dye species we cannot obtain the absorption anisotropy values. A negative value of $\left\langle\mathrm{P}_{4}\right\rangle$ usually suggests the occurrence of different species of the dye which can suggest that the number of types of dye species is higher than the number of the components of decay times. This is possible that the analysis of decay components is, to some extent, a formal mathematical procedure.

\section{RESULTS AND DISCUSSION}

\section{Absorption, fluorescence emission and fluorescence excitation spectra of dyes in PVA}

The shapes of the absorption spectra of uncoloured isotropic and anisotropic films are similar (Fig. 2), the differences in the absorption values are due to the change of film thickness. After normalization to the same thickness the spectra are similar. It was confirmed experimentally that in the excitation region studied the PVA emission (background) is low compared to the pigmented PVA films, and can be neglected.

Figure 3 shows the absorption, fluorescence emission and fluorescence excitation spectra of Mero A in PVA films. The shapes of the absorption spectra measured for unstretched and stretched films are similar (Fig. 3A). The film stretching practically does

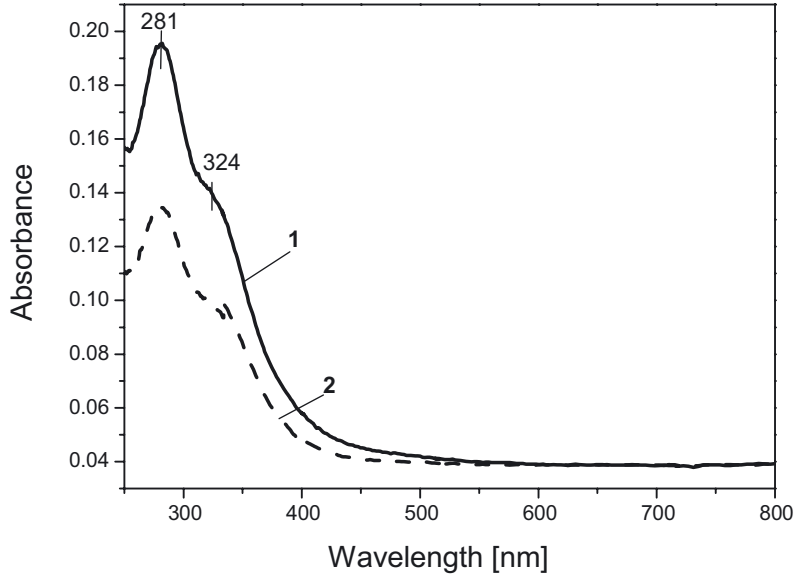

Figure 2. Absorption spectra of isotropic (1) and anisotropic (2) uncoloured PVA film measured in non-polarized light.

not change the shape of the absorption spectrum measured in non-polarized light (Fig. 3A). The decrease in absorption is due to the change in the film thickness. After normalization to the same thickness the spectra are very similar, suggesting that similar dye species occur in isotropic and anisotropic films. Mero A samples contain predominantly two species of the dye with absorption maxima at about $436 \mathrm{~nm}$ and $474 \mathrm{~nm}$ (Fig. 3A) and one band of emission at $546 \mathrm{~nm}$ being partially a result of excitation energy transfer between these species (Fig. 3B).

The maximum of the fluorescence excitation spectrum is located at about $513 \mathrm{~nm}$ (Fig. 3A), showing that a predominantly long wavelength species of Mero dye, practically not seen in the absorption spectrum, is responsible for the fluorescence. The maximum of the fluorescence emission for unstretched PVA film with Mero A was observed at

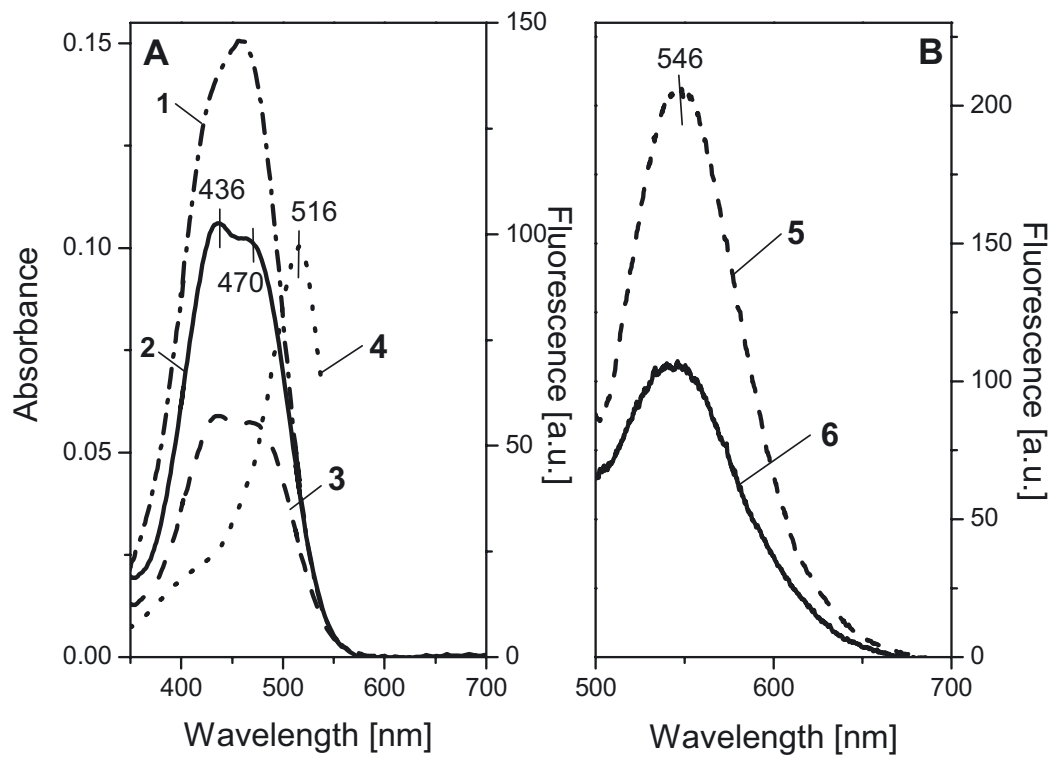

Figure 3. Absorption, fluorescence excitation and emission spectra of Mero A.

Spectra of (1-3) absorption; (4) fluorescence excitation (observed at $\left.\lambda_{\text {obs }}=600 \mathrm{~nm}\right)$ and $(5,6)$ fluorescence emission (excited at $\lambda_{\text {exc }}$ $=470 \mathrm{~nm}$ (5), $\lambda_{\text {exc }}=436 \mathrm{~nm}$ (6)) recorded in methanol solution (1), isotropic (2, 4-6) and anisotropic (3) PVA film. 


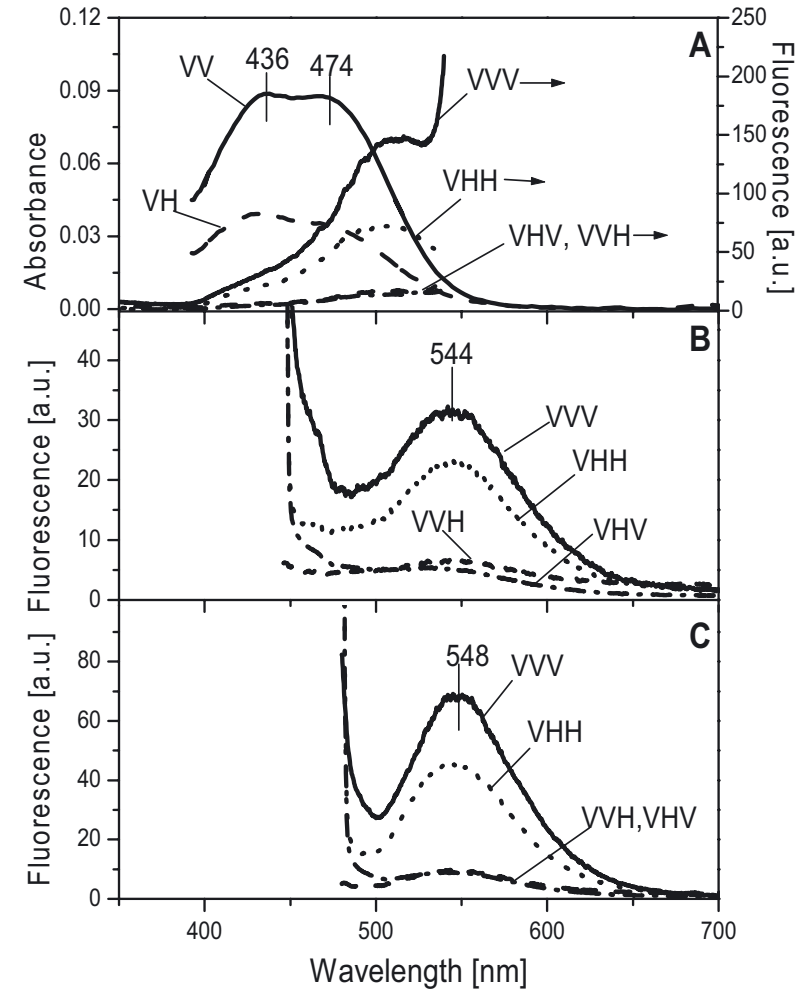

Figure 4. Polarized spectra of Mero A in anisotropic PVA film.

Spectra of (A) absorption and fluorescence excitation $\left(\lambda_{\mathrm{obs}}\right.$ $=550 \mathrm{~nm}) ;(\mathrm{B}, \mathrm{C})$ fluorescence emission recorded at $\lambda_{\text {exc }}=$ $436 \mathrm{~nm}(\mathrm{~B}), \lambda_{\mathrm{exc}}=470 \mathrm{~nm}(\mathrm{C})$, where $\mathrm{H}$, horizontal; $\mathrm{V}$, vertical, notation of polarized components (see Materials and Methods).

$546 \mathrm{~nm}$ (Fig. 3B), and showed that excitation at 470 $\mathrm{nm}$ was more effective than that at $436 \mathrm{~nm}$. In the latter case, the excitation energy should be transferred to the fluorescent species but some part of the excitation would be lost to heat (Staśkowiak et al., 2004).

Figure 4 shows the absorption, fluorescence excitation and emission spectra of Mero A, in stretched film measured in polarized light. The VVV and VHH emission components, at both wavelengths of excitation, are different, whereas the VVH and VHV components are both very low and similar in intensities (Fig. 4B and 4C). This shows that in most cases the TMs of dye emission and absorption form rather small angles with the direction of the film stretching. The anisotropies of emission coefficients calculated at the maximum of the emission band $\left(\lambda_{\mathrm{F}}\right)$ are shown in Table 1. From Fig. 4 and Table 1 it follows that Mero A is to a high degree oriented in the stretched film, which is confirmed by the high value of $S$ (Table 1) calculated from the absorption spectra (Fig. 4A).

A similar set of spectra for Mero B is presented in Figs. 5 and 6. For Mero B the situation is more complex than for Mero A. This dye, probably because of its elongated structure and its ionic form, can interact with the polymer differently and is able to form at least three spectral species. However, its fluorescence excitation spectrum shows that the 540$547 \mathrm{~nm}$ species is responsible for the fluorescence. The fluorescence spectra of the dye molecules consist of different bands. The polarized fluorescence spectra and anisotropy coefficients indicate that the emission TMs of the dyes are inclined at different angles to the film axis (Table 1). The dyes emitting at $414 \mathrm{~nm}$ have the emission TMs at a greater angle with the film axis than the TMs related to the dye molecules emitting at $460 \mathrm{~nm}$ and $512 \mathrm{~nm}$. The absorption TMs of the dyes are located at different angles to the film axis (Fig. 6A, Table 1), indicating that the energy transfer (ET) between the dye species is probably inefficient.

The PVA orientation parameter (S) calculated on the basis of the polarized absorption spectra at $283 \mathrm{~nm}$ (Fig. 2) is similar to that for Mero B at 461 $\mathrm{nm}$ and lower than that of other species of Mero A and B (Table 1). It is possible that the absorption TM of PVA is not exactly parallel to the stretching direction or that not all PVA molecules are highly oriented. In the latter case it is possible that most of the dye molecules interact preferentially with the welloriented PVA chains.

Figures 3 and 5 show that some of the dye molecules are oriented in the stretched PVA, but the anisotropy of their absorption and emission is different (Table 1). Anisotropy of fluorescence (Table 1) depends on the wavelength of excitation, which indicates the presence of different dye species whose orientation is probably affected by different interactions with the PVA matrix. Of course the VVV components usually have the highest intensity (the exception is the species at $414 \mathrm{~nm}$ of Mero B showing

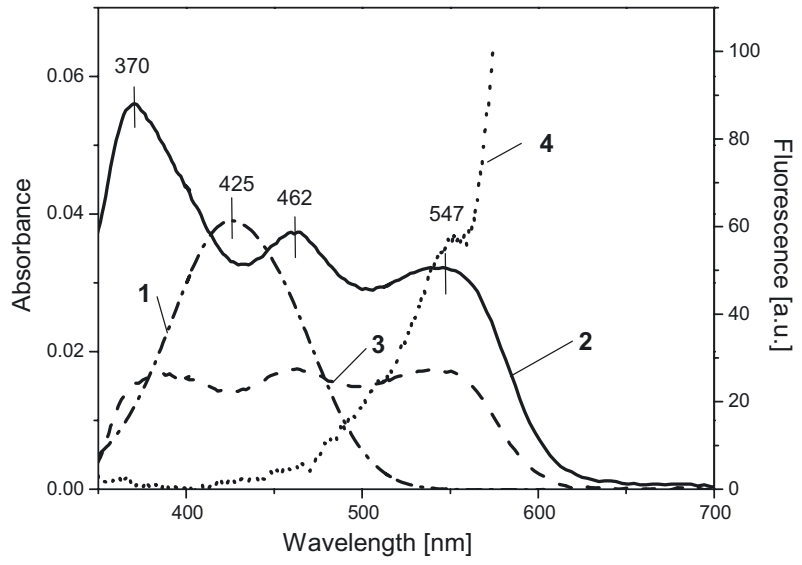

Figure 5. Absorption and fluorescence excitation $\left(\lambda_{\text {obs }}=\right.$ $600 \mathrm{~nm}$ ) spectra of Mero B.

Dye in methanol solution (1), isotropic (2), and anisotropic $(3,4)$ PVA film. 
Table 1. Orientation degree and anisotropy coefficients of Mero dyes in stretched PVA film.

\begin{tabular}{llllllllll}
\hline Mero & $\lambda_{\mathrm{A}}[\mathrm{nm}]$ & $\mathrm{S}$ & $\Theta\left[^{\circ}\right]$ & $\lambda_{\text {exc }}[\mathrm{nm}]$ & $\lambda_{\mathrm{F}}[\mathrm{nm}]$ & $\mathrm{r}_{\mathrm{a}}$ & $\mathrm{r}_{\mathrm{b}}$ & $\mathrm{r}_{\mathrm{c}}$ & $\mathrm{r}_{\mathrm{d}}$ \\
\hline \multirow{2}{*}{$\mathrm{A}$} & 436 & 0.23 & 45.7 & 436 & \multirow{2}{*}{544} & 0.54 & -0.35 & 0.63 & -0.31 \\
& 474 & 0.32 & 42.3 & 470 & & 0.69 & -0.37 & 0.69 & -0.37 \\
\hline \multirow{2}{*}{$\mathrm{B}$} & 461 & 0.09 & 51.2 & & 414 & 0.29 & 0.37 & -0.10 & -0.03 \\
& \multirow{2}{*}{540} & \multirow{2}{*}{0.22} & \multirow{2}{*}{46.1} & \multirow{2}{*}{370} & 460 & 0.53 & 0.08 & 0.21 & -0.19 \\
& & & & & 512 & 0.47 & 0.15 & 0.33 & 0.01 \\
\hline
\end{tabular}

S, degree of orientation (Eqn. 1) calculated from absorption; $r_{a^{\prime}}, r_{b^{\prime}} r_{c^{\prime}} r_{d^{\prime}}$ anisotropy coefficients (Eqns. 2-5) calculated from emission spectra; $\lambda_{\mathrm{A}^{\prime}} \lambda_{\mathrm{F}^{\prime}}$ the wavelength of absorption and fluorescence band, respectively; $\lambda_{\text {exc }^{\prime}}$ the wavelength of excitation; $\Theta$, the angle between transition moments of absorption and the stretching direction of the polymer film.

that the TMs of emission of some fractions of the dyes are oriented almost perpendicular to the direction of film stretching).

\section{Analysis of fluorescence decay}

Examples of measured and analyzed fluorescence decays of the VVV components obtained for Mero A and Mero B are shown in Fig. 7. Similar analyses were conducted for all measured samples and the obtained amplitudes and lifetimes of various decay components are presented in Tables 2 and 3. The coefficients of anisotropy (Eqns. 2-5 and 11,

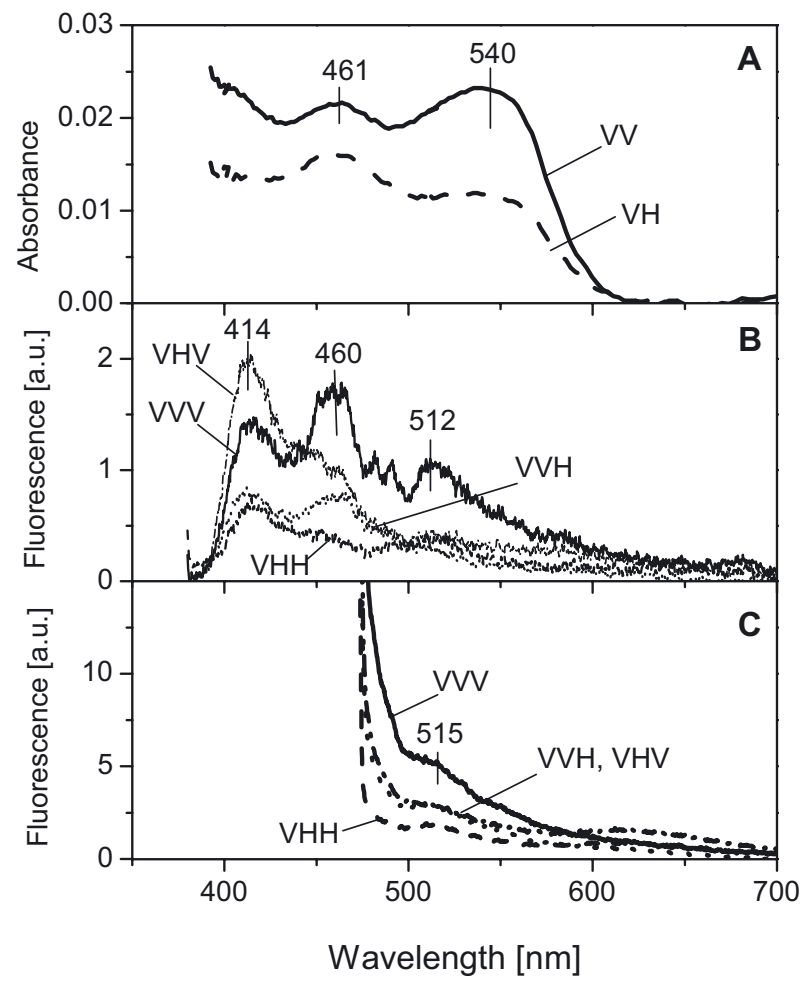

Figure 6. Polarized spectra of Mero B in anisotropic PVA film.

Spectra of (A) absorption, and (B, C) fluorescence emission: (B) $\lambda_{\text {exc }}=370 \mathrm{~nm},(\mathrm{C}) \lambda_{\text {exc }}=462 \mathrm{~nm}$, where $\mathrm{H}$, horizontal; $\mathrm{V}$, vertical; notation of polarized components (see Materials and Methods).
12) were also calculated from the components of fluorescence decay (Tables 4 and 5). On the basis of anisotropy coefficients and Legendre polynomials (Eqns. 13, 14, Tables 4 and 5) it is possible to obtain some quantitative information about the distribution of the long axis of elongated molecules, such as those of Mero A and Mero B, relative to the film orientation axis.

The results of the decay analysis obtained for Mero A in unstretched and stretched films are shown in Table 2. For decay measurements, the excitation at $470 \mathrm{~nm}$ was used. This makes the situation simpler than that for the excitation at 440 $\mathrm{nm}$ as the ET, responsible for the emission depolarization, occurs between at least two species of the dye. Observations were carried out at $546 \mathrm{~nm}$, near the maximum of the fluorescence band of this dye (Table 2). As follows from Table 2, in the film with Mero A, the intensities of the V0V and VVV components of fluorescence decay are the highest among the components obtained. There are differences between the results for the $\mathrm{VOV}$ and $\mathrm{VOH}$ components, not only in the intensity of emission but also in the number of components. However, the averaged lifetimes are not so different, which shows that it is possible to obtain more information from the decay components than from the averaged lifetimes of the sample. The unstretched film is practically isotropic, therefore the differences observed are related to those between the emission of promptly excited molecules in the V0V components and the emission of molecules predominantly excited by ET in $\mathrm{VOH}$. The excitation energy is transferred from molecules with the TMs of absorption almost parallel to the electric vector of the exciting light to the dye molecules oriented at a great angle with respect to the $\mathrm{V}$ direction. Migration of excitation takes time, therefore the intensity-weighted for the average lifetime in $\mathrm{VOV}$ is higher than in $\mathrm{VOH}$. The amplitude-weighted average lifetimes exhibit the opposite dependence at $470 \mathrm{~nm}$ excitation, but there also the number of components is different: three in $\mathrm{VOV}$ and only two in $\mathrm{VOH}$. This suggests that different species of the dye take part in ET 

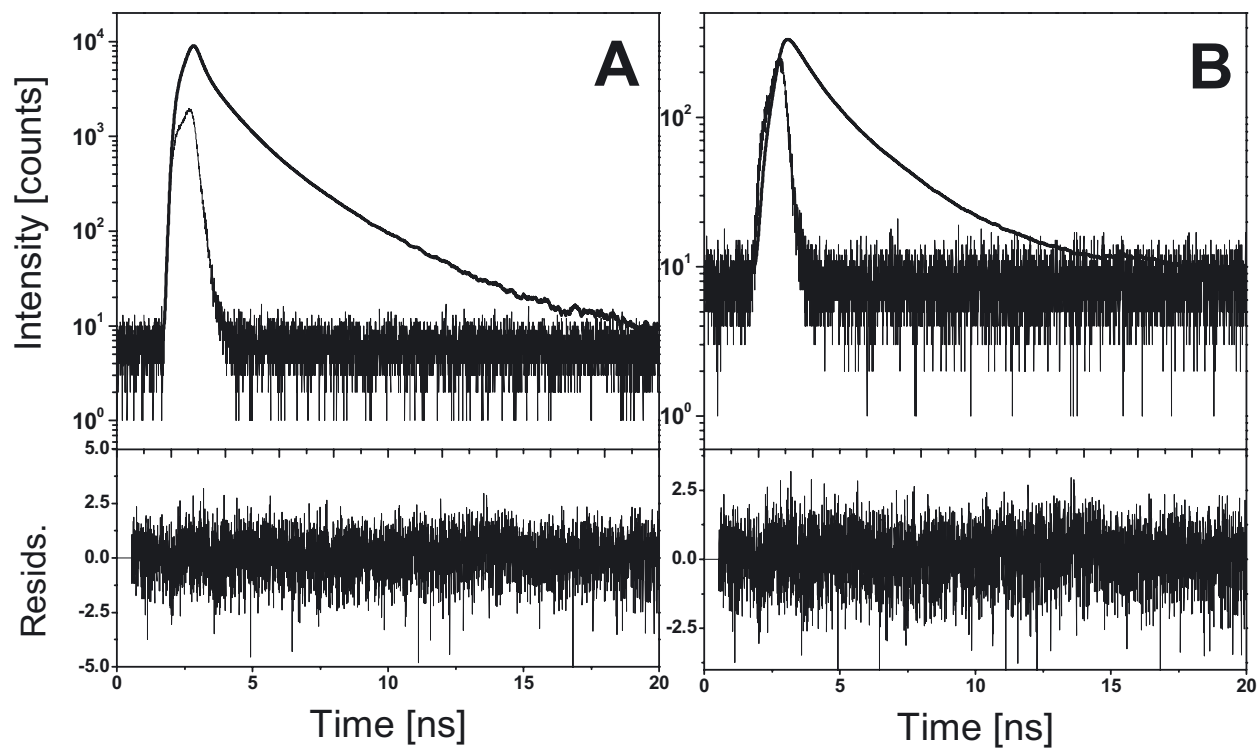

Figure 7. Examples of measured and analyzed fluorescence decays of VVV components.

(A) Mero A, $\lambda_{\text {exc }}=440 \mathrm{~nm}, \lambda_{\text {obs }}=546 \mathrm{~nm}, \alpha_{1}=(47960 \pm 950), \tau_{1}=(0.202 \pm 0.004) \mathrm{ns} ; \quad \alpha_{2}=(139509 \pm 220), \tau_{2}=(1.08 \pm 0.01)$ ns; $\alpha_{3}=(2990 \pm 75), \tau_{3}=(2.73 \pm 0.04) \mathrm{ns}$; (B) Mero B, $\lambda_{\text {exc }}=510 \mathrm{~nm} \lambda_{\text {obs }}=603 \mathrm{~nm}, \alpha_{1}=(1520 \pm 130), \tau_{1}=(0.72 \pm 0.06) \mathrm{ns} ; \alpha_{2}=$ $(1030 \pm 43), \tau_{2}=(2.64 \pm 0.09) \mathrm{ns}$.

with different efficiency. In this situation it is not easy to compare the amplitudes $\left(\alpha_{\mathrm{i}}\right)$ of the components and their decay times $\left(\tau_{\mathrm{i}}\right)$ (Table 2). This suggests that different dye species contribute to the emission observed.

The VOV component is greater than the $\mathrm{VOH}$ one, and VVV is greater than VVH, which shows that the angles between the TMs of absorption and emission are small. This was expected because of the elongated shape of the dye molecules. The rather high values of VHV compared to $\mathrm{VHH}$ suggest that not all molecules are perfectly oriented and their
TMs can make large angles with respect to the film axis.

A multi-exponential analysis of the decay is to some extent arbitrary, but from the number of the components obtained it is possible to draw conclusions about the presence of various fractions of molecules exhibiting different interactions with polymers. This shows that film stretching caused the dye orientation. The number of the decay components depends also on the wavelength of the excitation, suggesting that different species absorb and emit in different spectral regions.

Table 2. Fluorescence decay analysis of Mero A in unstretched and stretched PVA films.

\begin{tabular}{|c|c|c|c|c|c|c|c|c|c|c|c|c|c|c|c|c|c|}
\hline$\lambda_{\text {exc }}$ & $\lambda_{\mathrm{obs}}$ & \multirow{2}{*}{ Comp. } & \multicolumn{2}{|l|}{$\alpha_{1}$} & \multirow{2}{*}{$\begin{array}{l}\tau_{1} \\
{[\mathrm{~ns}]}\end{array}$} & \multicolumn{2}{|l|}{$\alpha_{2}$} & \multirow{2}{*}{$\begin{array}{c}\tau_{2} \\
{[\mathrm{~ns}]}\end{array}$} & \multicolumn{2}{|l|}{$\alpha_{3}$} & \multirow{2}{*}{$\begin{array}{l}\tau_{3} \\
{[n s]}\end{array}$} & \multicolumn{2}{|l|}{$\alpha_{4}$} & \multirow{2}{*}{$\begin{array}{l}\tau_{4} \\
{[\mathrm{~ns}]}\end{array}$} & \multirow{2}{*}{$\begin{array}{c}\tau_{\mathrm{AVI}} \\
{[\mathrm{ns}]}\end{array}$} & \multirow{2}{*}{$\frac{\tau_{\text {AVA }}}{[\mathrm{ns}]}$} & \multirow{2}{*}{$x^{2}$} \\
\hline$[\mathrm{nm}]$ & {$[\mathrm{nm}]$} & & [Cnts] & [\%] & & [Cnts] & {$[\%]$} & & [Cnts] & {$[\%]$} & & [Cnts] & [\%] & & & & \\
\hline \multirow{6}{*}{440} & \multirow{6}{*}{546} & V0V & 34230 & 74.25 & 0.21 & 10160 & 22.04 & 1.21 & 1710 & 3.71 & 3.40 & - & - & - & 1.42 & 0.55 & 1.038 \\
\hline & & $\mathrm{VOH}$ & 11750 & 76.87 & 0.15 & 2920 & 19.10 & 1.05 & 616 & 4.03 & 3.10 & - & - & - & 1.38 & 0.45 & 1.012 \\
\hline & & VVV & 47960 & 73.89 & 0.20 & 13950 & 21.50 & 1.08 & 2990 & 4.61 & 2.73 & - & - & - & 1.23 & 0.51 & 1.156 \\
\hline & & VVH & 7310 & 75.62 & 0.14 & 1803 & 18.67 & 0.83 & 552 & 5.71 & 2.47 & - & - & - & 1.23 & 0.40 & 0.958 \\
\hline & & VHV & 16370 & 77.91 & 0.12 & 3600 & 17.15 & 0.86 & 1038 & 4.94 & 2.47 & - & - & - & 1.21 & 0.36 & 1.025 \\
\hline & & VHH & 8480 & 78.14 & 0.12 & 1859 & 17.12 & 0.89 & 514 & 4.74 & 2.88 & - & - & - & 1.42 & 0.38 & 0.930 \\
\hline \multirow{6}{*}{470} & \multirow{6}{*}{546} & V0V & 11220 & 72.00 & 0.15 & 3437 & 22.06 & 0.82 & 927 & 5.95 & 2.39 & - & - & - & 1.17 & 0.43 & 1.003 \\
\hline & & $\mathrm{VOH}$ & 2212 & 81.00 & 0.27 & 519 & 19.00 & 1.67 & - & - & - & - & - & - & 1.10 & 0.53 & 1.073 \\
\hline & & VVV & 33840 & 59.77 & 0.15 & 14700 & 25.97 & 0.62 & 7824 & 13.82 & 1.76 & 255 & 0.45 & 4.46 & 1.23 & 0.51 & 1.089 \\
\hline & & VVH & 4900 & 69.24 & 0.11 & 1584 & 22.37 & 0.62 & 594 & 8.39 & 1.99 & - & - & - & 1.13 & 0.38 & 0.975 \\
\hline & & VHV & 5200 & 65.04 & 0.19 & 2108 & 26.36 & 0.88 & 688 & 8.60 & 2.29 & - & - & - & 1.24 & 0.55 & 1.003 \\
\hline & & VHH & 4870 & 70.83 & 0.11 & 1516 & 22.05 & 0.67 & 489 & 7.11 & 2.24 & - & - & - & 1.21 & 0.38 & 1.005 \\
\hline
\end{tabular}

Components (Comp.) notation: first letter, polarization of excited light; last one, polarization of observed emission; middle, 0 (isotropic, unstretched film) or orientation of sample axis (anisotropic, stretched sample); where V, vertical; $\mathrm{H}$, horizontal; $\tau_{\mathrm{AVI}^{\prime}} \tau_{\mathrm{AVA}^{\prime}}$ intensityweighted and amplitude-weighted estimated for the average lifetime; $\lambda_{\text {exc }} \lambda_{\text {obs' }}$ excitation and observation wavelengths; $\chi^{2}$, reduced chi square value; the accuracy of determined parameters is about $3-4 \%$. 
Table 3. Fluorescence decay analysis of Mero B in unstretched and stretched PVA films.

\begin{tabular}{|c|c|c|c|c|c|c|c|c|c|c|c|c|c|c|}
\hline$\lambda_{\text {exc }}$ & $\lambda_{\text {obs }}$ & \multirow{2}{*}{ Comp. } & \multicolumn{2}{|l|}{$\alpha_{1}$} & \multirow{2}{*}{$\frac{\tau_{1}}{[\mathrm{~ns}]}$} & \multicolumn{2}{|l|}{$\alpha_{2}$} & \multirow{2}{*}{$\frac{\tau_{2}}{[\mathrm{~ns}]}$} & \multicolumn{2}{|l|}{$\alpha_{3}$} & \multirow{2}{*}{$\begin{array}{l}\tau_{3} \\
{[\mathrm{~ns}]}\end{array}$} & \multirow{2}{*}{$\frac{\tau_{\mathrm{AVI}}}{[\mathrm{ns}]}$} & \multirow{2}{*}{$\begin{array}{l}\tau_{\mathrm{AVA}} \\
{[\mathrm{ns}]}\end{array}$} & \multirow{2}{*}{$\chi^{2}$} \\
\hline [nm] & {$[\mathrm{nm}]$} & & [Cnts] & [\%] & & [Cnts] & [\%] & & [Cnts] & [\%] & & & & \\
\hline \multirow{6}{*}{370} & \multirow{6}{*}{450} & V0V & 3810 & 53.58 & 0.78 & 2900 & 40.73 & 2.84 & 401 & 5.64 & 9.66 & 4.19 & 2.12 & 0.996 \\
\hline & & $\mathrm{VOH}$ & 1313 & 50.39 & 0.62 & 1096 & 42.05 & 2.37 & 197 & 7.56 & 8.07 & 3.90 & 1.91 & 0.933 \\
\hline & & VVV & 4970 & 52.03 & 0.82 & 4061 & 42.55 & 2.85 & 517 & 5.42 & 9.75 & 4.14 & 2.17 & 0.953 \\
\hline & & VVH & 1259 & 79.63 & 1.64 & 322 & 20.37 & 6.60 & - & - & - & 4.15 & 2.65 & 0.960 \\
\hline & & VHV & 1490 & 80.23 & 1.60 & 367 & 19.77 & 6.51 & - & - & - & 4.06 & 2.57 & 1.017 \\
\hline & & VHH & 1719 & 79.15 & 1.78 & 453 & 20.85 & 6.93 & - & - & - & 4.39 & 2.85 & 1.020 \\
\hline \multirow{6}{*}{370} & \multirow{6}{*}{513} & V0V & 1233 & 51.55 & 0.54 & 950 & 39.73 & 2.41 & 209 & 8.72 & 8.36 & 4.35 & 1.97 & 0.913 \\
\hline & & $\mathrm{VOH}$ & 637 & 75.96 & 0.99 & 202 & 24.04 & 5.35 & - & - & - & 3.74 & 2.04 & 1.004 \\
\hline & & VVV & 3270 & 54.25 & 0.63 & 2385 & 39.60 & 2.76 & 370 & 6.15 & 10.03 & 4.60 & 2.05 & 0.977 \\
\hline & & VVH & 811 & 76.73 & 1.48 & 246 & 23.27 & 6.83 & - & - & - & 4.60 & 2.73 & 1.014 \\
\hline & & VHV & 792 & 76.66 & 1.53 & 241 & 23.34 & 6.87 & - & - & - & 4.61 & 2.77 & 0.994 \\
\hline & & VHH & 946 & 75.45 & 1.78 & 308 & 24.55 & 7.44 & - & - & - & 5.04 & 3.17 & 1.009 \\
\hline \multirow{2}{*}{470} & \multirow{2}{*}{513} & V0V & 320 & 63.74 & 0.97 & 182 & 36.26 & 3.86 & - & - & - & 2.97 & 2.02 & 0.912 \\
\hline & & $\mathrm{VOH}$ & 117 & 61.46 & 0.90 & 73 & 38.54 & 3.57 & - & - & - & 2.80 & 1.93 & 0.860 \\
\hline \multirow{6}{*}{510} & \multirow{6}{*}{603} & V0V & 1950 & 58.96 & 0.81 & 1357 & 41.04 & 2.65 & - & - & - & 2.08 & 1.56 & 0.980 \\
\hline & & $\mathrm{VOH}$ & 449 & 48.88 & 0.58 & 469 & 51.12 & 2.27 & - & - & - & 1.93 & 1.44 & 0.926 \\
\hline & & VVV & 1520 & 59.60 & 0.72 & 1030 & 40.40 & 2.64 & - & - & - & 2.09 & 1.50 & 0.945 \\
\hline & & VVH & 453 & 63.17 & 0.80 & 264 & 36.83 & 2.37 & - & - & - & 1.79 & 1.38 & 0.917 \\
\hline & & VHV & 882 & 68.90 & 0.90 & 398 & 31.10 & 2.51 & - & - & - & 1.80 & 1.40 & 0.924 \\
\hline & & VHH & 773 & 57.17 & 0.78 & 579 & 42.83 & 2.12 & - & - & - & 1.68 & 1.35 & 0.933 \\
\hline
\end{tabular}

Description and components notation as in Table 2; the accuracy of determined parameters is about $3-4 \%$.

The average lifetimes in isotropic and anisotropic films are similar but these values are obtained by averaging the contributions from various fractions of different dye species. The information on these species can be obtained from the components of the fluorescence decay.

The data obtained for Mero B in isotropic and anisotropic films are shown in Table 3. From this table it is clear that most properties of both dyes (Mero A and Mero B) are similar, but for Mero B the $\mathrm{VHH}$ components are in some cases higher than the VHV ones. This shows that ET between differently oriented MeroB molecules is not as efficient as that for Mero A.
The numbers of polarized decay components for stretch and unstretched films are also different and depend on the wavelength of excitation. The latter dependence shows that different species of Mero B dye absorb and emit in different spectral regions. As follows from Table 4, the fraction of molecules almost perfectly oriented is higher for Mero A than for Mero B.

\section{Anisotropy of various components of decay}

The values of the coefficients of emission anisotropy obtained for the decay components of both dyes embedded in isotropic and anisotropic films are

Table 4. Anisotropy coefficient and Legendre polynomials calculated for emission decay components of Mero dyes in stretched PVA film.

\begin{tabular}{|c|c|c|c|c|c|c|c|c|c|c|c|c|c|c|}
\hline \multirow{2}{*}{ Mero } & \multirow{2}{*}{$\begin{array}{l}\lambda_{\text {exc }} \\
{[\mathrm{nm}]}\end{array}$} & \multirow{2}{*}{$\begin{array}{l}\lambda_{\text {obs }} \\
{[\mathrm{nm}]}\end{array}$} & \multicolumn{4}{|l|}{$\alpha_{1}$} & \multicolumn{4}{|l|}{$\alpha_{2}$} & \multicolumn{4}{|l|}{$\alpha_{3}$} \\
\hline & & & $\mathrm{r}_{\mathrm{a}}$ & $r_{b}$ & $r_{c}$ & $r_{d}$ & $\mathrm{r}_{\mathrm{a}}$ & $r_{b}$ & $r_{c}$ & $r_{d}$ & $\mathrm{r}_{\mathrm{a}}$ & $r_{b}$ & $r_{c}$ & $r_{d}$ \\
\hline \multirow{2}{*}{ A } & 440 & 546 & 0.65 & 0.24 & 0.39 & 0.05 & 0.69 & 0.24 & 0.49 & -0.01 & 0.60 & 0.25 & 0.39 & 0.02 \\
\hline & 470 & 546 & 0.66 & 0.02 & 0.65 & 0.002 & 0.73 & 0.12 & 0.67 & 0.02 & 0.80 & 0.12 & 0.78 & 0.07 \\
\hline \multirow{3}{*}{ B } & \multirow{2}{*}{370} & 450 & - & -0.05 & - & -0.10 & - & -0.07 & - & -0.11 & - & - & - & - \\
\hline & & 513 & - & -0.06 & - & -0.05 & - & -0.08 & - & -0.07 & - & - & - & - \\
\hline & 510 & 603 & 0.44 & 0.45 & 0.19 & -0.16 & 0.49 & -0.12 & 0.35 & -0.22 & - & - & - & - \\
\hline \multirow{3}{*}{ A } & & & $R_{a}$ & $\mathrm{R}_{\mathrm{b}}$ & $<\mathrm{P}_{2}>$ & $\left\langle\mathrm{P}_{4}\right\rangle$ & $R_{a}$ & $R_{b}$ & $<\mathrm{P}_{2}>$ & $\left\langle\mathrm{P}_{4}>\right.$ & $R_{a}$ & $R_{b}$ & $<\mathrm{P}_{2}>$ & $<\mathrm{P}_{4}>$ \\
\hline & 440 & 546 & 0.29 & -0.13 & 0.30 & -0.38 & 0.29 & -0.17 & 0.23 & -0.29 & 0.24 & 0.10 & 0.25 & -0.42 \\
\hline & 470 & 546 & 0.11 & -0.36 & 0.35 & -0.75 & 0.09 & -0.36 & 0.34 & -0.77 & - & - & - & - \\
\hline \multirow{3}{*}{ B } & \multirow{2}{*}{370} & 450 & 0.11 & -0.31 & 0.32 & -0.71 & 0.56 & -0.30 & 0.56 & -0.10 & - & - & - & - \\
\hline & & 513 & 0.27 & -0.23 & 0.35 & -0.48 & 0.26 & -0.38 & 0.44 & -0.58 & - & - & - & - \\
\hline & 510 & 603 & 0.08 & -0.33 & 0.32 & -0.75 & 0.10 & -0.34 & 0.36 & -0.74 & - & - & - & - \\
\hline
\end{tabular}

$\mathrm{r}_{\mathrm{a}^{\prime}} \mathrm{r}_{\mathrm{b}^{\prime}} \mathrm{r}_{\mathrm{c}^{\prime}} \mathrm{r}_{\mathrm{d}^{\prime}} \mathrm{R}_{\mathrm{a}^{\prime}} \mathrm{R}_{\mathrm{b}^{\prime}}$ anisotropy coefficient (Eqns. 2-5, 11 and 12), $<\mathrm{P}_{2}>,<\mathrm{P}_{4}>$, Legendre polynomials (Eqns. 13 and 14). 
Table 5. Anisotropy coefficient and Legendre polynomials calculated for amplitude-weighed of the average lifetime of Mero dyes in stretched PVA film.

\begin{tabular}{lllllllllll}
\hline Mero & $\begin{array}{c}\lambda_{\text {exc }} \\
{[\mathrm{nm}]}\end{array}$ & $\begin{array}{c}\lambda_{\text {obs }} \\
{[\mathrm{nm}]}\end{array}$ & $\mathrm{r}_{\mathrm{a}}$ & $\mathrm{r}_{\mathrm{b}}$ & $\mathrm{r}_{\mathrm{c}}$ & $\mathrm{r}_{\mathrm{d}}$ & $\mathrm{R}_{\mathrm{a}}$ & $\mathrm{R}_{\mathrm{b}}$ & $\left\langle\mathrm{P}_{2}\right\rangle$ & $\left\langle\mathrm{P}_{4}\right\rangle$ \\
\hline \multirow{2}{*}{$\mathrm{A}$} & 440 & 546 & -0.08 & 0.02 & -0.11 & -0.02 & -0.01 & 0.09 & 0.03 & -0.44 \\
& 470 & 546 & -0.09 & -0.12 & 0.03 & 0.00 & -0.15 & -0.17 & 0.14 & -0.79 \\
\hline \multirow{2}{*}{$\mathrm{B}$} & 370 & 450 & 0.07 & 0.04 & 0.06 & 0.03 & 0.11 & 0.07 & 0.08 & -0.40 \\
& & 513 & 0.10 & 0.05 & 0.11 & 0.05 & 0.09 & 0.03 & 0.10 & -0.44 \\
& 510 & 603 & -0.03 & -0.01 & -0.02 & -0.01 & 0.00 & 0.02 & 0.08 & -0.51 \\
\hline
\end{tabular}

Descriptions as in Table 4.

shown in Table 4. As follows from Table 4, various species are represented by three or two decay components of different orientations. This shows that the orientation influences the dye-polymer interaction.

For both species of Mero $\mathrm{A}$ the $\left\langle\mathrm{P}_{2}\right\rangle$ values given in Table 4 are similar to the $S$ values (Table 1). From the coefficients of emission anisotropy it follows that the fraction of well-oriented molecules is quite large. Significant differences are observed in $\left\langle\mathrm{P}_{2}>\right.$ between the $\alpha_{1}$ and $\alpha_{2}$ components for Mero B. This shows that the two groups of molecules described by these two exponentials are differently distributed. The value of $\left\langle\mathrm{P}_{4}>\right.$ for both groups is negative and changes irregularly with excitation wavelengths, which suggests that the observed species are characterized by different orientations.

Steady-state anisotropies of emission give much less information on the dye interactions with the polymer than the intensity of the polarized components decays. The values of anisotropy of Mero A obtained from average amplitude (Table 5) show a similar tendency as the values obtained from the absorption anisotropy (Table 1), but for Mero B the situation is much more complex, in agreement with the spectral properties of Mero B in PVA which are significantly different than those observed for this dye in solution (Staśkowiak et al., 2004).

The purpose of the present work was to study the fluorescence emitted from oriented dye molecules. We tried to qualify the dependence of the anisotropy coefficient values obtained from the polarized steady-state emission spectra and the polarized decay fluorescence components on the molecular array of the Mero dyes in polymer. As follows from Tables 1-5 and Figs. 1-7 as well as from a comparison of the data with the results obtained for the same dyes in isotropic media (Staśkowiak et al., 2004), the interaction of the dye with polymer chains has a strong influence on the anisotropy of the dye absorption and emission as well as in some cases even on the shape of the polarized emission spectra.
Mero dyes can be easily oriented in anisotropic systems, hence they are useful for the investigation of the biological cell elements (e.g. membranes) stained by the dye.

The interactions with polymer lead to formation of species with different emission spectra in Mero B, a merocyanine in the salt form, whereas for Mero A differently oriented molecules exhibit various decay times (it means also various yields of emission). The results presented confirm that the interactions of the dyes investigated depend on the dye properties such as the chain length or/and the salt form of the molecule, and influence the dye orientation with respect to the elongated polymer macromolecules.

Although the analysis of the decays of the polarized fluorescence components is only a formal procedure, it shows that the fractions of molecules characterized by different times of fluorescence decay exhibit different anisotropy of emission. The anisotropy coefficients should be taken into account when evaluating the efficiency of introduction of sensitizer molecules into cells. Such evaluation is carried out very often only on the basis of the fluorescence of the dye in the cells. It has been shown that the overall fluorescence quantum yield of 540-merocyanine can change with the lipid to Mero dye ratio in model systems and that the Mero fluorescence efficiency increases in highly packed lipid interface (Bernik et al., 1999). The fluorescent band of 540-merocyanine has also been used to monitor lipid-phase domains in studies of tumour cells (Stillwell et al., 1993). The results show that the selection of proper sensitizers for PDT treatment cannot be done only on the basis of fluorescence measurements. Dye interactions with biological molecules can change the fluorescence yield and may also influence the yield of triplet states generation by intersystem crossing. The triplet states are very active in photochemical reactions, and are therefore important in photodynamic treatment. The photoinactivation effect of 540-merocyanine on artificial and natural cell membranes involves singlet 
molecular oxygen as well as the singlet oxygen effects on plasma membrane integrity and mitochondrial respiration (Gaffney et al., 1990; Kalyanaraman et al., 1987).

Mero dyes seem to be promising optical sensors with spectral properties, including the calculated anisotropy coefficients, sensitive to the molecular environment, useful to study anisotropic, artificial and biological, membranes. Their orientation and interactions with neighbouring molecules in membrane influence the photophysical and photochemical parameters of the dye-sensitizers. The strong interactions with the cell membrane can prevent efficient penetration of the sensitizer molecules into cells and can therefore disturb PDT, but strong interactions with macromolecules in pathological cells enhance the photodynamic effects. Therefore, further investigation of dye interactions with cancerous and healthy cells and their components is necessary.

\section{Acknowledgements}

This work was supported by the Ministry of Education and Science (Poland), grant No. 2 P05D 04630 (2006-2009) (to A.D., D.F.), and by Texas Emerging Technologies Fund Grant (to I.G., Z.G.) and by Poznań University of Technology (to E.T.).

\section{REFERENCES}

Anderson GS, Miyagi K, Sampson RW, Sieber F (2002) Anti-tumor effect of Merocyanine 540-mediated photochemotherapy combined with Edelfosine: potential implications for the ex vivo purging of hematopoietic stem cell grafts from breast cancer patients. J Photochem Photobiol B:Biol 68: 101-108.

Bernik D, Tymyczyszyn E, Daraio ME, Negri RM (1999) Fluorescent dimers of merocyanine 540 (MC540) in the gel phase of phosphatidylcholine liposomes. Photochem Photobiol 70: 40-48.

Bojarski P, Gryczyński I, Kułak L, Synak A, Burnett A (2006) Excitation energy migration between elongated fluorophores in uniaxially oriented polyvinyl alcohol films. Chem Phys Lett 431: 94-99.

Bucci E, Gryczyński Z, Fronticelli C, Gryczyński I, Lakowicz JR (1992) Fluorescence intensity and anisotropy decays of the intrinsic tryptophan emission of hemoglobin measured with a $10-\mathrm{GHz}$ fluorometer using front-face geometry on a free liquid surface. J Fluorescence 2: 29-35.

Dalmolen LGP, Picken SJ, de Jong AF, de Jeu WH (1985) The order parameters $\left\langle\mathrm{P}_{2}>\right.$ and $\left\langle\mathrm{P}_{4}>\right.$ in nematic p-alkylp'-cyanobiphenyls: polarized Raman measurements and the influence of molecular association. J Phys 46: 1443-1449.

Frąckowiak D, Gruda I, Niedbalska M, Romanowski M, Dudkowiak A (1990) Polarized absorption and emission spectra of stilbazolium merocyanines. J Photochem Photobiol A:Chem 54: 37-48.

Frąckowiak D, Wiktorowicz K, Cofta J, Niedbalska M, Latosińska M (1995) Incorporation of stilbazolium merocyanines into resting and stimulated mononuclear leukocytes. Acta Biochim Polon 42: 61-68.

Frąckowiak D, Ptak A, Gryczynski Z, Gryczynski I, Targowski P, Zelent B (2004) Fluorescence polarization studies of B-phycoerythrin oriented in polymer film. Photochem Photobiol 79: 11-20.

Gaffney DK, Schober SL, Sieber F (1990) Merocyanine 540sensitized photoinactivation of leukemia cells: role of oxygen and effects on plasma membrane integrity and mitochondrial respiration. Exp Hematol 18: 23-26.

Gruda I, Page M, Bolduc F, Lariberte St, Noel Ch (1987) Merocyanines: potential new anticancer agents. Anticancer Res 7: 1125-1128.

Gryczyński Z, Fronticelli C, Tenenholz T, Bucci E (1993) Effect of disordered hemes on energy transfer rates between tryptophans and heme in myoglobin. Biophys $J$ 65: 1951-1958.

Kalyanaraman B, Feix JB, Sieber F, Thomas JP, Girotti AW (1987) Photodynamic action of merocyanine 540 on artificial and natural cell membranes: involvement of singlet molecular oxygen. Proc Natl Acad Sci USA 84: 2999-3003.

Lakowicz JR (1999) Principles of fluorescence spectroscopy. 2 edn, Kluwer Academic, Plenum Publishers, New York, Boston, Dordrecht, London, Moscow.

Lakowicz JR, Gryczyński I (1991) Frequency domain fluorescence spectroscopy. In Topics in Fluorescence Spectroscopy (Lakowicz JR, eds) vol 1, pp 394-335. Plenum Press, New York.

Lydaki E, Dimitriou H, Papazoglou Th, Bolonaki E, Kalmanti M (1996) Merocyanine 540 mediated photoirradiation of leukemic cells. In vitro influence on cell survival. J Photochem Photobiol B:Biol 32: 27-32.

Mishra A, Behera RK, Behera PK, Mishra BK, Behera GB (2000) Cyanines during the 1990s: A review. Chem Rev 100: 1793-2011.

O’Brien MJ, Gaffney DK, Wang TP, Seibel F (1992) Merocyanine 540 sensitized photoinactivation of envelope viruses in blood products: site and mechanism of phototoxicity. Blood 80: 277-285.

Smith OM, Gaffney DK, Anderson MS, McOlash L, Schober SL, Sieber F (1991) Plasma membrane properties regulating the sensitivity of leukemia, lymphoma and solid tumor cells to merocyanine 540 sensitized photoirradiation. Exp Hematol 19: 785-788.

Staśkowiak E, Dudkowiak A, Hanyż I, Wiktorowicz K, Frąckowiak D (2004) Spectral properties of stilbazolium merocyanines - potential sensitizers in photodynamic therapy and diagnosis. Part I. Merocyanines in model systems. J Photochem Photobiol A:Chem 163: 127-134.

Staśkowiak E, Dudkowiak A, Wiktorowicz K, Cofta J, Frąckowiak D (2005) Spectral properties of stilbazolium merocyanines - potential sensitizers in photodynamic therapy and diagnosis. Part II. Merocyanines in resting and stimulated lymphocytes. I Photochem Photobiol A: Chem 169: 159-168.

Stillwell W, Wassall SR, Dumaval AC, Ehringen DW, Browning CW, Jenski LJ (1993) Use of merocyanine (MC540) in quantifying lipid domains and packing in phospholipid vesicles and tumor cells. Biochim Biophys Acta 1146: $135-144$.

Wiktorowicz K, Niedbalska M, Planner A, Frąckowiak D (1995) Incorporation of stilbazolium merocyanines into human leukocytes measured by flow cytometry. Acta Biochim Polon 42: 333-338.

Wiktorowicz K, Cofta J, Dudkowiak A, Waszkowiak A, Frąckowiak D (2004) Preliminary studies of phthalocyanine sensitizers incorporated into human leukemia cells from two cell-lines. Acta Biochim Polon 51: 703-710. 